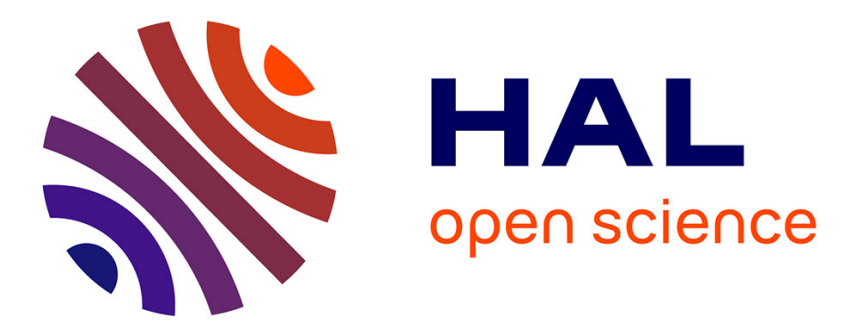

\title{
Linear response theory for thermally driven quantum open systems
}

\author{
Vojkan Jaksic, Yoshiko Ogata, Claude-Alain Pillet
}

\section{To cite this version:}

Vojkan Jaksic, Yoshiko Ogata, Claude-Alain Pillet. Linear response theory for thermally driven quantum open systems. Journal of Statistical Physics, 2006, 123 (3), pp.547-569. 10.1007/s10955006-9075-1 . hal-00009009v2

\section{HAL Id: hal-00009009 \\ https://hal.science/hal-00009009v2}

Submitted on 7 Feb 2006

HAL is a multi-disciplinary open access archive for the deposit and dissemination of scientific research documents, whether they are published or not. The documents may come from teaching and research institutions in France or abroad, or from public or private research centers.
L'archive ouverte pluridisciplinaire HAL, est destinée au dépôt et à la diffusion de documents scientifiques de niveau recherche, publiés ou non, émanant des établissements d'enseignement et de recherche français ou étrangers, des laboratoires publics ou privés. 


\title{
Linear response theory for thermally driven quantum open systems
}

\author{
V. Jakšić ${ }^{1}$, Y. Ogata ${ }^{2,3}$, C.-A. Pillet ${ }^{2}$ \\ ${ }^{1}$ Department of Mathematics and Statistics \\ McGill University \\ 805 Sherbrooke Street West \\ Montreal, QC, H3A 2K6, Canada \\ ${ }^{2}$ CPT-CNRS, UMR 6207 \\ Université du Sud, Toulon-Var, B.P. 20132 \\ F-83957 La Garde Cedex, France \\ ${ }^{3}$ Department of Mathematical Sciences \\ University of Tokyo \\ Komaba,Tokyo,153-8914 Japan
}

February 7, 2006

Dedicated to Barry Simon on the occasion of his 60th birthday

\begin{abstract}
This note is a continuation of our recent paper [JOP1] where we have proven the Green-Kubo formula and the Onsager reciprocity relations for heat fluxes in thermally driven quantum open systems. In this note we extend the derivation of the Green-Kubo formula to heat and charge fluxes and discuss some other generalizations of the model and results of [JOP1].
\end{abstract}




\section{Introduction}

This paper is the second in a series dedicated to linear response theory for non equilibrium steady states (NESS) of quantum open systems. The development of linear response theory is a part of a much wider research program initiated in [Ru1, Ru2, Ru3, JP1, JP2, JP3]. This program deals with mathematical foundations of non-equilibrium thermodynamics in the framework of algebraic quantum statistical mechanics. Motivated by the developments in classical non-equilibrium statistical mechanics (see the review [Ru4]), the program addresses the central issue of NESS in two independent steps.

(A) The existence and analytic properties of NESS are assumed as an axiom. On the basis of this axiom one develops the mathematical theory of non-equilibrium quantum statistical mechanics in an abstract setting. This step is primarily concerned with the mathematical structure of the theory and its relation to the fundamental physical aspects of non-equilibrium (see [DGM, KTH]).

(B) The second step concerns study of specific physically relevant models. Relaxation to a NESS and analytical properties of this NESS are detailed dynamical problems which can be answered only in the context of concrete models. Once these fundamental problems are solved, the thermodynamics and the transport theory of the model are derived from the general structural results established in (A).

So far, the main focus of the program has been the second law of thermodynamics (positivity of the entropy production). In this case the part (A) has been settled in [Ru2, JP1, JP4], where the entropy production has been defined in the abstract framework of algebraic quantum statistical mechanics. In these works various structural properties of the entropy production have been established and in particular it was shown that the entropy production of any NESS is non-negative. The strict positivity of the entropy production is a problem which belongs to the category (B). At the moment there are two classes of non-trivial models whose NESS are well-understood and which have strictly positive entropy production. The first class of models describes an $N$-level quantum system coupled to finitely many independent free Fermi gas reservoirs [Da, LeSp, JP2]. The second class describes finitely many free Fermi gas reservoirs coupled by local interactions [BM, AM, FMU]. Some exactly solvable spin or fermion models with strictly positive entropy production have been studied in [AH, AP, AJPP1, AJPP2].

The natural next step in this program is the development of linear response theory and in particular the derivation of the Green-Kubo formulas (abbreviated GKF). A typical physical situation we consider concerns the steady states of a quantum device, a confined system $\mathcal{S}$ with a finite number of degrees of freedom, coupled to $M$ reservoirs $\mathcal{R}_{1}, \ldots, \mathcal{R}_{M}$, see Figure 1 (a generalization of this setup is discussed in Section 5). More specifically, we are interested in situations where the system $\mathcal{S}$ is driven out of equilibrium by thermodynamic forces, i.e., by discrepancies in the intensive thermodynamic parameters of the reservoirs around some common equilibrium values. Suppose that each reservoir $\mathcal{R}_{j}$ is in a thermal equilibrium state characterized by some inverse temperature $\beta_{j}=\beta-X_{j}$ and chemical potential $\mu_{j}=\mu+Y_{j} / \beta$. If some of the forces $X_{j}, Y_{j}$ do not vanish, then under normal conditions they induce energy and mass/charge currents across the system $\mathcal{S}$. Linear response theory is concerned with the calculation of these currents to first order in the forces. In [JOP1] we have derived the GKF for heat fluxes (the case $\mu=Y_{j}=0$ ) in the axiomatic framework of algebraic quantum statistical mechanics. In this note we discuss a derivation which applies to both heat and charge fluxes and complete the step (A) of the program. Concerning (B), the examples to which our derivation directly applies include all models for which the strict positivity of the entropy production has been established. These applications are discussed in the forthcoming papers [JOP2, JOP3, JOPP].

In classical mechanics there is a number of different ways to describe an open system out of thermal equilibrium. Some of these descriptions involve various kinds of thermostating devices which lead to non-Hamiltonian effective equations of motion (see [EM, RB]). Due to the intrinsic Hamiltonian nature of quantum dynamics, the situation is different for quantum open systems. Except in some special limiting cases (e.g., in the weak coupling limit, see [LeSp]) one is forced to consider the joint dynamics of the system $\mathcal{S}$ and its environment.

To describe the joint system $\mathcal{S}+\mathcal{R}_{1}+\cdots+\mathcal{R}_{M}$ we suppose that it in inially prepared in a state where each reservoir $\mathcal{R}_{j}$ is characterized by intensive thermodynamic parameters $\beta_{j}$ and $\mu_{j}$. Due to the interactions between the system $\mathcal{S}$ and the reservoirs this state is not stationary. We shall assume that, as $t \rightarrow+\infty$, the joint system relaxes to a steady state. Since confined quantum systems have discrete spectrum and almost periodic dynamics, a 


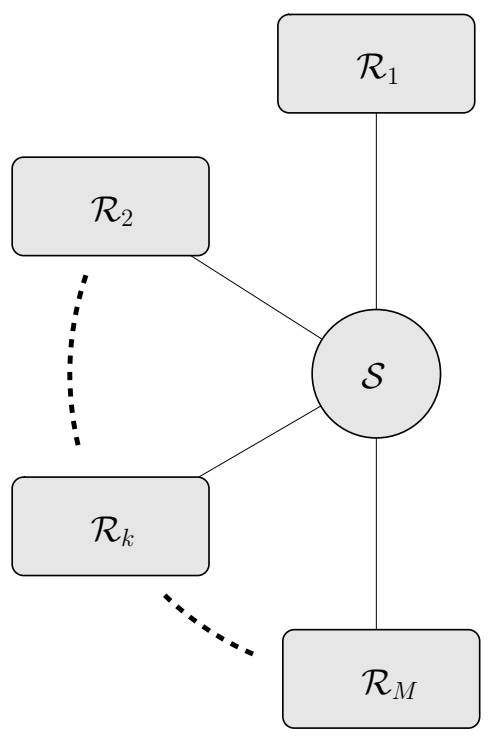

Figure 1: An open system with $M$ reservoirs.

non-trivial steady state may exist only if the reservoirs are infinitely extended. Moreover, in order for this steady state to be a NESS (i.e., to avoid the joint system to relax to an equilibrium state), the reservoirs must be "ideal" in the following sense. A reservoir serves two purposes: on the one hand it is a source feeding energy/particles to the device $\mathcal{S}$ in a statistically controlled way. On the other hand it also works as a sink or dissipator, transporting to spatial infinity energy/particles coming out of $\mathcal{S}$. In an "ideal" reservoir the source and the sink are independent as much as quantum dynamics allows. The fact that incoming and outgoing fluxes do not interact ensures that the intensive thermodynamic parameters describing the initial state of an "ideal" reservoir still apply to the outgoing flux in a steady state. Thus, "ideal" reservoirs are able to maintain fluxes across the system $\mathcal{S}$ over the infinite time interval needed to reach a steady state.

Linear response theory of NESS is a delicate interplay between three limits which must be taken in a definite order. First, one must perform the thermodynamic (or infinite volume) limit of the reservoirs. Then, a $t \rightarrow+\infty$ limit is necessary to reach a NESS. Final $X_{j}, Y_{j} \rightarrow 0$ limits are needed to extract the linear response proper.

For interacting quantum systems the first limit is already a difficult problem which can only be treated in a limited number of models (see e.g. Chapter 6 in [BR2]). However, for the ideal reservoirs we are dealing with, this problem is well understood (see e.g. Section 5.2 in [BR2]). An infinitely extended quantum dynamical system at non-vanishing density can be described in the universal conceptual framework of algebraic quantum statistical mechanics. It is therefore possible to decouple the thermodynamic limit from the two remaining ones. In this paper we derive the GKF under the assumption that the these two limits exist and can be interchanged. The justification of this fact is a delicate dynamical problem which belongs to the category (B) and will be treated in the aforementioned companion papers.

This note is organized as follows. For notational purposes, in Section 2 we quickly review a few basic notions of algebraic quantum statistical mechanics. In Section 3 we introduce the model and review basic concepts of nonequilibrium statistical mechanics (the reader may complement this section with reviews [JP3, AJPP1]). Linear response theory is discussed in Section 4. Our main result is stated in Subsection 4.2. Its proof follows closely the arguments in [JOP1] and is outlined in Subsection 4.3. Various generalizations of our model and results are discussed in Section 5.

Acknowledgment. The research of V.J. was partly supported by NSERC. A part of this work has been done during the visit of V.J. to CPT-CNRS. Y.O. is supported by the Japan Society for the Promotion of Science. This work has 
been done during the stay of Y.O. to CPT-CNRS, partly supported by the Canon Foundation in Europe and JSPS.

\section{Basic notions}

Let $\mathcal{O}$ be a $C^{*}$-algebra with identity $\mathbb{1}$ and $\tau^{t}, t \in \mathbb{R}$, a strongly continuous group of $*$-automorphisms of $\mathcal{O}$. The group $\tau$ and the pair $(\mathcal{O}, \tau)$ are often called $C^{*}$-dynamics and $C^{*}$-dynamical system. A state $\omega$ on $\mathcal{O}$ is called $\tau$ invariant if $\omega \circ \tau^{t}=\omega$ for all $t \in \mathbb{R}$. An anti-linear involutive $*$-automorphism $\Theta: \mathcal{O} \rightarrow \mathcal{O}$ is called time-reversal of $(\mathcal{O}, \tau)$ if $\Theta \circ \tau^{t}=\tau^{-t} \circ \Theta$ for all $t \in \mathbb{R}$. A state $\omega$ on $\mathcal{O}$ is called time-reversal invariant if $\omega(\Theta(A))=\omega\left(A^{*}\right)$ for all $A \in \mathcal{O}$.

We call quantum dynamical system a triple $(\mathcal{O}, \tau, \omega)$ where $\omega$ is a given state on $\mathcal{O}$. The state $\omega$ describes the initial (or reference) thermodynamical state of the system and is not necessarily $\tau$-invariant (for a discussion of this point we refer the reader to Section 2 of [AJPP1]). Under normal conditions, i.e., under natural ergodicity assumptions, all $\omega$-normal states are thermodynamically equivalent reference states in the sense that they lead to the same NESS.

We denote by $\operatorname{Ent}\left(\eta_{1} \mid \eta_{2}\right)$ the Araki relative entropy of two states $\eta_{1}$ and $\eta_{2}$. We use the sign and ordering convention of [BR2, Don, DJP] (hence, $\operatorname{Ent}\left(\eta_{1} \mid \eta_{2}\right) \in[-\infty, 0]$ ). The Araki relative entropy has played an important role in recent developments in non-equilibrium quantum statistical mechanics.

Let $\beta>0$. A state $\omega$ is called a $(\tau, \beta)$-KMS state if for all $A, B \in \mathcal{O}$ there exists a function $F_{A, B}(z)$, analytic in the strip $S_{\beta}=\{z \in \mathbb{C} \mid 0<\operatorname{Im} z<\beta\}$, bounded and continuous on its closure, and satisfying the KMS-boundary condition

$$
F_{A, B}(t)=\omega\left(A \tau^{t}(B)\right), \quad F_{A, B}(t+\mathrm{i} \beta)=\omega\left(\tau^{t}(B) A\right) .
$$

As usual, we write $\omega\left(A \tau^{z}(B)\right)=F_{A, B}(z)$ for $z \in \bar{S}_{\beta}$ even when $\tau^{z}(B)$ is not well-defined. A $(\tau, \beta)$-KMS states describes a physical system in thermal equilibrium at inverse temperature $\beta$. For all practical purposes these states can be considered as thermodynamic limits of Gibbs canonical ensembles.

The general theory of chemical potential in quantum statistical mechanics is discussed in Section 5.4.3 of [BR2]. In our study of linear response theory we will only consider the chemical potential associated to the usual $U(1)$ gauge invariance of quantum mechanics. We will call charge flux the current associated to the corresponding conserved charge. The extension of our results to more general gauge groups is straightforward. Since we only need a fraction of the mathematical structures commonly associated to the chemical potential we shall be brief. Let $\vartheta^{\varphi}$ be a $C^{*}$-dynamics on $\mathcal{O}$ such that $\tau^{t} \circ \vartheta^{\varphi}=\vartheta^{\varphi} \circ \tau^{t}$ for all $t, \varphi \in \mathbb{R}$. $\vartheta$ is the gauge-group and its elements $\vartheta^{\varphi}$ are gauge transformations. Physical observables are invariant under gauge transformations and are therefore elements of

$$
\mathcal{O}_{\vartheta}=\left\{A \in \mathcal{O} \mid \vartheta^{\varphi}(A)=A \text { for all } \varphi \in \mathbb{R}\right\} .
$$

Note that $\mathcal{O}_{\vartheta}$ is a $\tau$-invariant $C^{*}$-subalgebra of $\mathcal{O}$ and so $\left(\mathcal{O}_{\vartheta}, \tau\right)$ is a $C^{*}$-dynamical system. Let $\mu \in \mathbb{R}$ and

$$
\alpha^{t}=\tau^{t} \circ \vartheta^{-\mu t}
$$

Clearly $\tau^{t}$ and $\alpha^{t}$ coincide on $\mathcal{O}_{\vartheta}$. We say that a state $\omega$ on $\mathcal{O}$ is a $(\tau, \vartheta, \beta, \mu)$-KMS state if it is an $(\alpha, \beta)$-KMS state. Although this last terminology is not common, it is convenient for our purposes. A $(\tau, \vartheta, \beta, \mu)$-KMS state describes a physical system in equilibrium at inverse temperature $\beta$ and chemical potential $\mu$. Note that if $\omega$ is a $(\tau, \vartheta, \beta, \mu)$-KMS state on $\mathcal{O}$, then its restriction to the gauge-invariant subalgebra $\mathcal{O}_{\vartheta}$ is a $(\tau, \beta)$-KMS state on $\mathcal{O}_{\vartheta}$ which describes a thermodynamic limit of grand canonical ensembles associated to the parameters $\beta, \mu$.

\section{The model and the framework}

\subsection{The model}

Our starting point are two $C^{*}$-dynamical systems $\left(\mathcal{O}_{\mathrm{L}}, \tau_{\mathrm{L}}\right)$ and $\left(\mathcal{O}_{\mathrm{R}}, \tau_{\mathrm{R}}\right)$ with gauge-groups $\vartheta_{\mathrm{L}}$ and $\vartheta_{\mathrm{R}}$. For convenience we shall call them the left, L, and the right, R, system. We denote the generators of $\tau_{\mathrm{L}}, \tau_{\mathrm{R}}, \vartheta_{\mathrm{L}}$ and $\vartheta_{\mathrm{R}}$ 
by $\delta_{\mathrm{L}}, \delta_{\mathrm{R}}, \xi_{\mathrm{L}}$ and $\xi_{\mathrm{R}}$. For many applications the left system can be thought of as composed of a first reservoir and a confined system, $\mathrm{L}=\mathcal{S}+\mathcal{R}_{1}$, while the right system is just a second reservoir, $\mathrm{R}=\mathcal{R}_{2}$. The generalizations of this setup are discussed in Section 5.

The $C^{*}$-algebra of the joint system $\mathrm{L}+\mathrm{R}$ is $\mathcal{O}=\mathcal{O}_{\mathrm{L}} \otimes \mathcal{O}_{\mathrm{R}}$ and its decoupled (non-interacting) dynamics is $\tau_{0}=\tau_{\mathrm{L}} \otimes \tau_{\mathrm{R}}$. The generator of $\tau_{0}$ is $\delta_{0}=\delta_{\mathrm{L}} \otimes I+I \otimes \delta_{\mathrm{R}}$. In the sequel, whenever the meaning is clear within the context, we shall write $\delta_{\mathrm{L}}$ for $\delta_{\mathrm{L}} \otimes I, \delta_{\mathrm{R}}$ for $I \otimes \delta_{\mathrm{R}}$, etc.

The gauge-group of the joint system is $\vartheta=\vartheta_{\mathrm{L}} \otimes \vartheta_{\mathrm{R}}$ and its generator is $\xi=\xi_{\mathrm{L}}+\xi_{\mathrm{R}}$. We denote by $\mathcal{O}_{\vartheta}$ the corresponding gauge-invariant subalgebra of $\mathcal{O}$.

Let $V \in \mathcal{O}_{\vartheta}$ be a self-adjoint element describing the interaction of $\mathrm{L}$ and $\mathrm{R}$. The interacting $C^{*}$-dynamics $\tau$ is generated by $\delta=\delta_{0}+\mathrm{i}[\mathrm{V}, \cdot]$ and commutes with the gauge-group $\vartheta$. The coupled (interacting) joint system $\mathrm{L}+\mathrm{R}$ is described by the $C^{*}$-dynamical system $(\mathcal{O}, \tau)$.

\subsection{The reference states}

We set $I_{\epsilon}(x)=(x-\epsilon, x+\epsilon)$ and write $I_{\epsilon}=I_{\epsilon}(0)$.

Let $\beta_{\mathrm{eq}}>0$ and $\mu_{\mathrm{eq}} \in \mathbb{R}$ be given reference (equilibrium) values of the inverse temperature and the chemical potential. We make the following assumptions concerning the initial states of $\mathrm{L}$ and $\mathrm{R}$.

(A1) $\omega_{\mathrm{L}}$ is the unique $\left(\tau_{\mathrm{L}}, \vartheta_{\mathrm{L}}, \beta_{\mathrm{eq}}, \mu_{\mathrm{eq}}\right)$-KMS state on $\mathcal{O}_{\mathrm{L}}$. The reference states of $\mathrm{R}$ are parametrized by $\beta \in I_{\epsilon_{1}}\left(\beta_{\text {eq }}\right)$ and $\mu \in I_{\epsilon_{2}}\left(\mu_{\text {eq }}\right)$ and $\omega_{\mathrm{R}, \beta, \mu}$ is the unique $\left(\tau_{\mathrm{R}}, \vartheta_{\mathrm{R}}, \beta, \mu\right)$-KMS state on $\mathcal{O}_{\mathrm{R}}$. We shall denote $\omega_{\mathrm{R}, \beta_{\mathrm{eq}}, \mu_{\mathrm{eq}}}$ by $\omega_{\mathrm{R}}$.

Throughout the paper we shall assume that (A1) holds. The reference states of our model are $\omega_{\mathrm{L}} \otimes \omega_{\mathrm{R}, \beta, \mu}$, $\beta \in I_{\epsilon_{1}}\left(\beta_{\mathrm{eq}}\right), \mu \in I_{\epsilon_{2}}\left(\mu_{\mathrm{eq}}\right)$. For our purposes it is convenient to introduce the parameters (thermodynamical forces)

$$
X=\beta_{\mathrm{eq}}-\beta, \quad Y=\beta \mu-\beta_{\mathrm{eq}} \mu_{\mathrm{eq}},
$$

and to parametrize the reference states by $X$ and $Y$, i.e., we write

$$
\omega_{X, Y, 0}=\omega_{\mathrm{L}} \otimes \omega_{\mathrm{R}, \beta, \mu}
$$

Since we are interested in linear response theory, without loss of generality we may restrict the values of $X, Y$ to $I_{\epsilon}$, where $\epsilon>0$ is a small positive number. Note that $\omega_{0,0,0}$ is the unique $\left(\tau_{0}, \vartheta, \beta_{\text {eq }}, \mu_{\text {eq }}\right)$-KMS state on $\mathcal{O}$.

As we have already mentioned, under normal conditions all $\omega_{X, Y, 0}$-normal states are thermodynamically equivalent reference states of $\mathrm{L}+\mathrm{R}$. We now describe a particular $\omega_{X, Y, 0}$-normal reference state which will play an important role in our discussion of linear response theory.

Set

$$
\alpha_{\mathrm{L}}^{t}=\tau_{\mathrm{L}}^{t} \circ \vartheta_{\mathrm{L}}^{-\mu_{\mathrm{eq}} t}, \quad \alpha_{\mathrm{R}, \mu}^{t}=\tau_{\mathrm{R}}^{t} \circ \vartheta_{\mathrm{R}}^{-\mu t} .
$$

Assumption (A1) implies that $\omega_{\mathrm{L}}$ is the unique $\left(\alpha_{\mathrm{L}}, \beta_{\mathrm{eq}}\right)$-KMS state on $\mathcal{O}_{\mathrm{L}}$ and that $\omega_{\mathrm{R}, \beta, \mu}$ is the unique $\left(\alpha_{\mathrm{R}, \mu}, \beta\right)$ KMS state on $\mathcal{O}_{\mathrm{R}}$. Set

$$
\alpha_{X, Y, 0}^{t}=\alpha_{\mathrm{L}}^{t} \otimes \alpha_{\mathrm{R}, \mu}^{\beta t / \beta_{\mathrm{eq}}} .
$$

Then $\omega_{X, Y, 0}$ is the unique $\left(\alpha_{X, Y, 0}, \beta_{\text {eq }}\right)$-KMS state on $\mathcal{O}$. Let $\delta_{X, Y, 0}$ be the generator of $\alpha_{X, Y, 0}$ and

$$
\delta_{X, Y}=\delta_{X, Y, 0}+\mathrm{i}[V, \cdot] .
$$

The subalgebra $\operatorname{Dom}\left(\delta_{\mathrm{L}}\right) \cap \operatorname{Dom}\left(\xi_{\mathrm{L}}\right) \cap \operatorname{Dom}\left(\delta_{\mathrm{R}}\right) \cap \operatorname{Dom}\left(\xi_{\mathrm{R}}\right)$ is a core for $\delta_{X, Y, 0}$ and $\delta_{X, Y}$. On this subalgebra $\delta_{X, Y, 0}$ acts as

$$
\delta_{X, Y, 0}=\delta_{0}-\mu_{\mathrm{eq}} \xi-\frac{X}{\beta_{\mathrm{eq}}} \delta_{\mathrm{R}}-\frac{Y}{\beta_{\mathrm{eq}}} \xi_{\mathrm{R}}
$$

Let $\alpha_{X, Y}$ be the $C^{*}$-dynamics generated by $\delta_{X, Y}$. Araki's perturbation theory yields that there exists a unique $\left(\alpha_{X, Y}, \beta_{\text {eq }}\right)$-KMS state $\omega_{X, Y}$ on $\mathcal{O}$. 
The states $\omega_{X, Y, 0}$ and $\omega_{X, Y}$ are mutually normal. The reference states $\omega_{X, Y}$ will play a central role in our study of linear response theory. Note that $\omega_{0,0}$ is the unique $\left(\tau, \vartheta, \beta_{\text {eq }}, \mu_{\text {eq }}\right)$-KMS state on $\mathcal{O}$. We denote this state by $\omega_{\text {eq }}$. The next assumption concerns the $\left(\tau, \beta_{\text {eq }}\right)$-KMS state induced by $\omega_{\text {eq }}$ on the gauge invariant subalgebra $\mathcal{O}_{\vartheta}$.

(A2) For all $A, B \in \mathcal{O}_{\vartheta}$,

$$
\lim _{|t| \rightarrow \infty} \omega_{\mathrm{eq}}\left(\tau^{t}(A) B\right)=\omega_{\mathrm{eq}}(A) \omega_{\mathrm{eq}}(B)
$$

A well-known consequence of the KMS condition and Assumption (A2) is the relation

$$
\lim _{t \rightarrow+\infty} \int_{-t}^{t} \omega_{\text {eq }}\left(\left[\tau^{s}(A), B\right]\right) \mathrm{d} s=0,
$$

which holds for all $A, B \in \mathcal{O}_{\vartheta}$ (see Theorem 5.4.12 in [BR2]). This relation plays a key role in the derivation of the Onsager reciprocity relations.

\subsection{Non-equilibrium steady states}

We postulate relaxation to a NESS as follows:

(A3) For all $X, Y \in I_{\epsilon}$ there exists a state $\omega_{X, Y,+}$ on $\mathcal{O}_{\vartheta}$ such that for all $A \in \mathcal{O}_{\vartheta}$,

$$
\lim _{t \rightarrow+\infty} \omega_{X, Y}\left(\tau^{t}(A)\right)=\omega_{X, Y,+}(A) .
$$

Assumptions (A2) and (A3) are strong ergodic hypotheses which are difficult to verify in concrete models. We remark that in typical physical situations one expects more, namely that

$$
\lim _{t \rightarrow+\infty} \eta\left(\tau^{t}(A)\right)=\omega_{X, Y,+}(A),
$$

for all $\omega_{X, Y, 0}$-normal states $\eta$ and $A \in \mathcal{O}_{\vartheta}$. Indeed, such strong form of approach to NESS has been established in all examples we consider in [JOP2, JOP3, JOPP]. However, we will not need such an assumption in our axiomatic study of linear response theory.

\subsection{Time-reversal invariance}

Our next assumption concerns time-reversal.

(A4) There exists a time-reversal $\Theta$ of $\left(\mathcal{O}, \tau_{0}\right)$ such that $\Theta(V)=V$ and

$$
\begin{aligned}
\Theta \circ \tau_{\mathrm{L}}^{t}=\tau_{\mathrm{L}}^{-t} \circ \Theta, & \Theta \circ \tau_{\mathrm{R}}^{t}=\tau_{\mathrm{R}}^{-t} \circ \Theta, \\
\Theta \circ \vartheta_{\mathrm{L}}^{\varphi}=\vartheta_{\mathrm{L}}^{-\varphi} \circ \Theta, & \Theta \circ \vartheta_{\mathrm{R}}^{\varphi}=\vartheta_{\mathrm{R}}^{-\varphi} \circ \Theta,
\end{aligned}
$$

for all $t, \varphi \in \mathbb{R}$.

Clearly, $\Theta$ is a time-reversal of $(\mathcal{O}, \vartheta)$ and $\left(\mathcal{O}, \alpha_{X, Y, 0}\right)$. In particular it leaves $\mathcal{O}_{\vartheta}$ invariant. It is not difficult to show that $\Theta$ is also a time-reversal of $(\mathcal{O}, \tau)$ and $\left(\mathcal{O}, \alpha_{X, Y}\right)$, and that the states $\omega_{X, Y, 0}$ and $\omega_{X, Y}$ are time-reversal invariant. The proofs of these facts are the same as the proof of Lemma 3.1 in [JOP1]. 


\subsection{Fluxes}

To define the flux observables we need:

(A5) $V \in \operatorname{Dom}\left(\delta_{\mathrm{R}}\right) \cap \operatorname{Dom}\left(\xi_{\mathrm{R}}\right)$.

If (A5) holds, we set

$$
\Phi=\delta_{\mathrm{R}}(V), \quad \mathcal{J}=\xi_{\mathrm{R}}(V) .
$$

The observable $\Phi$ describes the heat flux out of the system R. The observable $\mathcal{J}$ describes the charge flux out of R. Since $V \in \mathcal{O}_{\vartheta}$ and $\tau_{\mathrm{R}}, \vartheta_{\mathrm{R}}$ commute with $\vartheta$ we have $\Phi, \mathcal{J} \in \mathcal{O}_{\vartheta}$. If the time-reversal assumption (A4) holds, then

$$
\Theta(\Phi)=-\Phi, \quad \Theta(\mathcal{J})=-\mathcal{J}
$$

\subsection{Entropy balance equation}

In the recent literature the entropy balance equation has been always discussed with respect to the product reference state $\omega_{X, Y, 0}[\mathrm{Ru} 2, \mathrm{Ru} 3, \mathrm{JP} 1, \mathrm{JP} 3, \mathrm{JP} 4]$. The finite time entropy balance equation w.r.t. the reference state $\omega_{X, Y}$ has the following form.

Theorem 3.1 Assume that $V \in \operatorname{Dom}\left(\delta_{\mathrm{L}}\right) \cap \operatorname{Dom}\left(\xi_{\mathrm{L}}\right) \cap \operatorname{Dom}\left(\delta_{\mathrm{R}}\right) \cap \operatorname{Dom}\left(\xi_{\mathrm{R}}\right)$. Then

$$
\operatorname{Ent}\left(\omega_{X, Y} \circ \tau^{t} \mid \omega_{X, Y}\right)=-X \int_{0}^{t} \omega_{X, Y}\left(\tau^{s}(\Phi)\right) \mathrm{d} s-Y \int_{0}^{t} \omega_{X, Y}\left(\tau^{s}(\mathcal{J})\right) \mathrm{d} s .
$$

Proof. The assumptions of the theorem imply that $V \in \operatorname{Dom}\left(\delta_{X, Y}\right)$. Since $V \in \mathcal{O}_{\vartheta}$ implies $\xi(V)=0$, we have

$$
\beta_{\text {eq }} \delta_{X, Y}(V)=\beta_{\text {eq }} \delta(V)-X \Phi-Y \mathcal{J}
$$

The entropy balance equation of [JP1, JP4] yields

$$
\begin{aligned}
\operatorname{Ent}\left(\omega_{X, Y} \circ \tau^{t} \mid \omega_{X, Y, 0}\right) & =\operatorname{Ent}\left(\omega_{X, Y} \mid \omega_{X, Y, 0}\right)+\beta_{\mathrm{eq}} \int_{0}^{t} \omega_{X, Y}\left(\tau^{s}\left(\delta_{X, Y}(V)\right)\right) \mathrm{d} s \\
& =\operatorname{Ent}\left(\omega_{X, Y} \mid \omega_{X, Y, 0}\right)+\beta_{\mathrm{eq}} \omega_{X, Y}\left(\tau^{t}(V)\right)-\beta_{\mathrm{eq}} \omega_{X, Y}(V) \\
& -X \int_{0}^{t} \omega_{X, Y}\left(\tau^{s}(\Phi)\right) \mathrm{d} s-Y \int_{0}^{t} \omega_{X, Y}\left(\tau^{s}(\mathcal{J})\right) \mathrm{d} s .
\end{aligned}
$$

The fundamental formula of Araki [Ar1, Ar2] (see also [BR2, Don, DJP]) yields that

$$
\begin{aligned}
\operatorname{Ent}\left(\omega_{X, Y} \circ \tau^{t} \mid \omega_{X, Y}\right) & =\operatorname{Ent}\left(\omega_{X, Y} \circ \tau^{t} \mid \omega_{X, Y, 0}\right)-\beta_{\mathrm{eq}} \omega_{X, Y}\left(\tau^{t}(V)\right)+C, \\
\operatorname{Ent}\left(\omega_{X, Y} \mid \omega_{X, Y, 0}\right) & =\beta_{\mathrm{eq}} \omega_{X, Y}(V)-C,
\end{aligned}
$$

where $C$ is a constant expressible in terms of the modular structure (we do not need its explicit form here). The relations (3.6) and (3.7) yield the statement.

The entropy production of the NESS $\omega_{X, Y,+}$ is defined by

Theorem 3.1 yields

$$
\operatorname{Ep}\left(\omega_{X, Y,+}\right)=-\lim _{t \rightarrow+\infty} \frac{\operatorname{Ent}\left(\omega_{X, Y} \circ \tau^{t} \mid \omega_{X, Y}\right)}{t} .
$$

$$
\operatorname{Ep}\left(\omega_{X, Y,+}\right)=X \omega_{X, Y,+}(\Phi)+Y \omega_{X, Y,+}(\mathcal{J}) \geq 0,
$$

and this relation is the second law of thermodynamics for our model. Of course, if $(X, Y) \neq(0,0)$, then under normal conditions one expects that $\operatorname{Ep}\left(\omega_{X, Y,+}\right)>0$, i.e., that the fluxes are non-vanishing. The strict positivity of entropy production is a detailed dynamical question which can be answered only in the context of specific models. 


\subsection{Centered observables}

An observable $A \in \mathcal{O}$ is called centered if $\omega_{X, Y}(A)=0$ for all $X, Y \in I_{\epsilon}$. We denote by $\mathcal{C}$ the set of all centered observables. Obviously, $\mathcal{C}$ is a norm-closed vector subspace of $\mathcal{O}$. Our derivation of the Green-Kubo formula applies only to centered observables.

If Assumption (A4) holds, then any self-adjoint observable $A$ satisfying $\Theta(A)=-A$ is centered. Indeed, since $\omega_{X, Y}$ is time-reversal invariant,

$$
\omega_{X, Y}(A)=\omega_{X, Y}(\Theta(A))=-\omega_{X, Y}(A)
$$

and so $\omega_{X, Y}(A)=0$. In particular, if (A4) holds, then the flux observables $\Phi$ and $\mathcal{J}$ are centered.

It is an important fact that the flux observables are centered irrespectively of the time-reversal assumption. This fact will play a central role in our discussion of the Green-Kubo formula for systems which are not time-reversal invariant.

Proposition 3.2 Under Assumption (A5)

$$
\omega_{X, Y}(\Phi)=\omega_{X, Y}(\mathcal{J})=0
$$

holds for all $X, Y \in I_{\epsilon}$.

Proof. Assume first that

$$
V \in \operatorname{Dom}\left(\delta_{\mathrm{L}}\right) \cap \operatorname{Dom}\left(\xi_{\mathrm{L}}\right) \cap \operatorname{Dom}\left(\delta_{\mathrm{R}}\right) \cap \operatorname{Dom}\left(\xi_{\mathrm{R}}\right) .
$$

Note that $C^{*}$-dynamics $\alpha_{X, Y}$ is well-defined for all $X, Y \in \mathbb{R}$. The following generalization of the entropy balance equation (3.4) holds: for all $X, Y \in I_{\epsilon}$ and $Z, U \in \mathbb{R}$,

$$
\begin{aligned}
\operatorname{Ent}\left(\omega_{X, Y} \circ \alpha_{Z, U}^{t} \mid \omega_{X, Y}\right)= & -(X-Z) \int_{0}^{t} \omega_{X, Y}\left(\alpha_{Z, U}^{s}(\Phi)\right) \mathrm{d} s \\
& -(Y-U) \int_{0}^{t} \omega_{X, Y}\left(\alpha_{Z, U}^{s}(\mathcal{J})\right) \mathrm{d} s .
\end{aligned}
$$

The proof of this relation is essentially the same as the proof of (3.4). The only difference is that the relation (3.5) is now replaced with

$$
\beta_{\mathrm{eq}} \delta_{X, Y}(V)=\beta_{\mathrm{eq}} \delta_{Z, U}(V)-(X-Z) \Phi-(Y-U) \mathcal{J}
$$

The entropy balance equation of [JP1, JP4] yields

$$
\operatorname{Ent}\left(\omega_{X, Y} \circ \alpha_{Z, U}^{t} \mid \omega_{X, Y, 0}\right)=\operatorname{Ent}\left(\omega_{X, Y} \mid \omega_{X, Y, 0}\right)+\beta_{\mathrm{eq}} \int_{0}^{t} \omega_{X, Y}\left(\alpha_{Z, U}^{s}\left(\delta_{X, Y}(V)\right)\right) \mathrm{d} s,
$$

and the rest of the argument follows line by line the proof of Theorem 3.1.

The equation (3.10) yields

$$
\lim _{t \downarrow 0} \frac{\operatorname{Ent}\left(\omega_{X, Y} \circ \alpha_{Z, U}^{t} \mid \omega_{X, Y}\right)}{t}=-(X-Z) \omega_{X, Y}(\Phi)-(Y-U) \omega_{X, Y}(\mathcal{J}),
$$

and so for all $X, Y \in I_{\epsilon}$ and $Z, U \in \mathbb{R}$,

$$
(X-Z) \omega_{X, Y}(\Phi)+(Y-U) \omega_{X, Y}(\mathcal{J}) \geq 0 .
$$

This relation yields the statement. 
To prove the general case, let $V \in \operatorname{Dom}\left(\delta_{\mathrm{R}}\right) \cap \operatorname{Dom}\left(\xi_{\mathrm{R}}\right)$ and

$$
V_{j}=\frac{j}{\pi} \int_{\mathbb{R}^{2}} \mathrm{e}^{-j\left(t^{2}+s^{2}\right)} \tau_{\mathrm{L}}^{t} \circ \vartheta_{\mathrm{L}}^{s}(V) \mathrm{d} t \mathrm{~d} s, \quad j=1,2, \ldots
$$

The observables $V_{j}$ satisfy (3.9). Let $\omega_{X, Y, j}$ and $\Phi_{j}, \mathcal{J}_{j}$ be the reference state and the flux observables associated to $V_{j}$. We have established that for all $X, Y \in I_{\epsilon}$,

$$
\omega_{X, Y, j}\left(\Phi_{j}\right)=\omega_{X, Y, j}\left(\mathcal{J}_{j}\right)=0 .
$$

By the properties of analytic approximations (see [BR2]), $\left\|\omega_{X, Y, j}-\omega_{X, Y}\right\| \rightarrow 0,\left\|\Phi_{j}-\Phi\right\| \rightarrow 0,\left\|\mathcal{J}_{j}-\mathcal{J}\right\| \rightarrow 0$ as $j \rightarrow \infty$, and the statement follows from (3.12).

Note that we did not use the gauge invariance of $V$ in the above proof.

\subsection{Regular observables}

As mentioned in the introduction, our derivation of GKF relies on the assumption that the $t \rightarrow+\infty$ limit can be interchanged with differentiation w.r.t. $X, Y$. We note that if the states $\omega_{\mathrm{L}}, \omega_{\mathrm{R}, \beta, \mu}$ are ergodic for $\beta, \mu$ sufficiently close to $\beta_{\mathrm{eq}}, \mu_{\mathrm{eq}}$ then it is not difficult to show that the states $\omega_{X, Y}$ are mutually singular for distinct values of $X, Y$. Therefore the differentiability of the function $(X, Y) \mapsto \omega_{X, Y}\left(\tau^{t}(A)\right)$ is an extremely delicate question, already for finite $t$. However, as we shall see in Subsection 4.2, one can prove that this function is differentiable at $X=Y=0$ under very mild regularity assumptions on $A$, provided $A$ is centered (this is the content of our main technical result, Theorem 4.2).

The following definition encapsulates our assumption on the interchange of limits.

Definition 3.3 Assume that (A3) holds. Let $A \in \mathcal{O}_{\vartheta}$ be an observable such that the function

$$
(X, Y) \mapsto \omega_{X, Y}\left(\tau^{t}(A)\right),
$$

is differentiable at $(0,0)$ for all $t$. We call such an observable regular if the function

$$
(X, Y) \mapsto \omega_{X, Y,+}(A),
$$

is also differentiable at $(0,0)$ and

$$
\begin{gathered}
\left.\lim _{t \rightarrow+\infty} \partial_{X} \omega_{X, Y}\left(\tau^{t}(A)\right)\right|_{X=Y=0}=\left.\partial_{X} \omega_{X, Y,+}(A)\right|_{X=Y=0}, \\
\left.\lim _{t \rightarrow+\infty} \partial_{Y} \omega_{X, Y}\left(\tau^{t}(A)\right)\right|_{X=Y=0}=\left.\partial_{Y} \omega_{X, Y,+}(A)\right|_{X=Y=0}
\end{gathered}
$$

\section{Linear response theory}

\subsection{Overview}

Suppose that Assumptions (A3) and (A5) hold and that the functions

$$
(X, Y) \mapsto \omega_{X, Y,+}(\Phi), \quad(X, Y) \mapsto \omega_{X, Y,+}(\mathcal{J}),
$$


are differentiable at $(0,0)$. The kinetic transport coefficients are defined by

$$
\begin{aligned}
& L_{\mathrm{hh}}=\left.\partial_{X} \omega_{X, Y,+}(\Phi)\right|_{X=Y=0}, \\
& L_{\mathrm{hc}}=\left.\partial_{Y} \omega_{X, Y,+}(\Phi)\right|_{X=Y=0}, \\
& L_{\mathrm{ch}}=\left.\partial_{X} \omega_{X, Y,+}(\mathcal{J})\right|_{X=Y=0}, \\
& L_{\mathrm{cc}}=\left.\partial_{Y} \omega_{X, Y,+}(\mathcal{J})\right|_{X=Y=0},
\end{aligned}
$$

where indices h/c stand for heat/charge. Linear response theory is concerned with these coefficients. An elementary consequence of the second law (Relation (3.8)) is that the matrix

$$
L=\left[\begin{array}{ll}
L_{\mathrm{hh}} & L_{\mathrm{hc}} \\
L_{\mathrm{ch}} & L_{\mathrm{cc}}
\end{array}\right]
$$

is positive definite on the real vector space $\mathbb{R}^{2}$ (this of course does not imply that $L_{\mathrm{hc}}=L_{\mathrm{ch}}$ !).

The Green-Kubo formulas are at the center of linear response theory. For $A, B \in \mathcal{O}_{\vartheta}$ we set

$$
\mathcal{L}(A, B)=\lim _{t \rightarrow+\infty} \frac{1}{2} \int_{-t}^{t} \omega_{\mathrm{eq}}\left(A \tau^{s}(B)\right) \mathrm{d} s .
$$

The GKF assert that if the system is time-reversal invariant, then

$$
\begin{aligned}
& L_{\mathrm{hh}}=\mathcal{L}(\Phi, \Phi), \\
& L_{\mathrm{hc}}=\mathcal{L}(\Phi, \mathcal{J}), \\
& L_{\mathrm{ch}}=\mathcal{L}(\mathcal{J}, \Phi), \\
& L_{\mathrm{cc}}=\mathcal{L}(\mathcal{J}, \mathcal{J}) .
\end{aligned}
$$

These formulas are mathematical expressions of the fluctuation-dissipation mechanism in statistical mechanicsthey link linear response to a thermodynamical force to the equilibrium correlations w.r.t. the corresponding flux observable.

The coefficients $L_{\mathrm{hc}}$ and $L_{\mathrm{ch}}$ are of particular physical importance. In words, the chemical potential difference may cause a heat flow out of $\mathrm{R}$ even if $\mathrm{L}$ and $\mathrm{R}$ are at the same temperature. For $Y$ small, this flow is equal to $Y L_{\mathrm{hc}}+o(Y)$. Similarly, the temperature difference may cause a charge flow out of $\mathrm{R}$ even if $\mathrm{L}$ and $\mathrm{R}$ have equal chemical potentials. For $X$ small this flow is equal to $X L_{\mathrm{ch}}+o(X)$. An immediate consequence of the second and third relation in (4.15) and the formula (3.3) are the Onsager reciprocity relations

$$
L_{\mathrm{hc}}=L_{\mathrm{ch}} \text {. }
$$

For $A, B \in \mathcal{O}_{\vartheta}$ and $t \in \mathbb{R}$ we set

$$
\mathfrak{L}(A, B, t)=\frac{1}{\beta_{\text {eq }}} \int_{0}^{t} \mathrm{~d} s \int_{0}^{\beta_{\text {eq }}} \mathrm{d} u \omega_{\text {eq }}\left(\tau^{s}(A) \tau^{\mathrm{i} u}(B)\right),
$$

and

$$
\mathfrak{L}(A, B)=\lim _{t \rightarrow+\infty} \mathfrak{L}(A, B, t),
$$

whenever the limit exists. We remark that by the KMS condition the function

$$
(s, z) \mapsto \omega_{\mathrm{eq}}\left(\tau^{s}(A) \tau^{z}(B)\right),
$$


is bounded and continuous on the set $\mathbb{R} \times \bar{S}_{\beta_{\mathrm{eq}}}$. The central step in our derivation of (4.15) are the following formulas

$$
\begin{aligned}
& L_{\mathrm{hh}}=\mathfrak{L}(\Phi, \Phi), \\
& L_{\mathrm{hc}}=\mathfrak{L}(\Phi, \mathcal{J}), \\
& L_{\mathrm{ch}}=\mathfrak{L}(\mathcal{J}, \Phi), \\
& L_{\mathrm{cc}}=\mathfrak{L}(\mathcal{J}, \mathcal{J}) .
\end{aligned}
$$

It is an important point that these formulas hold without the time-reversal assumption - they are the Green-Kubo formulas for systems which are not time-reversal invariant. The Green-Kubo formulas (4.15) are an immediate consequence of (4.17) and the following result established in [JOP1].

Proposition 4.1 Suppose that Assumptions (A1), (A2), and (A4) hold and let $A, B \in \mathcal{O}_{\vartheta}$ be two self-adjoint observables which are both even or odd under $\Theta$. Then

$$
\mathcal{L}(A, B)=\mathfrak{L}(A, B) .
$$

Proof. The argument follows line by line the proof of Theorem 2.3 in [JOP1]. For reader convenience we outline the main steps of the argument.

We need to prove that

$$
\lim _{t \rightarrow+\infty} \frac{1}{\beta_{\mathrm{eq}}} \int_{0}^{\beta_{\mathrm{eq}}}\left[\int_{0}^{t} \omega_{\mathrm{eq}}\left(\tau^{s}(A) \tau^{\mathrm{i} u}(B)\right) \mathrm{d} s\right] \mathrm{d} u=\lim _{t \rightarrow+\infty} \int_{-t}^{t} \omega_{\mathrm{eq}}\left(A \tau^{s}(B)\right) \mathrm{d} s .
$$

The time-reversal invariance and the KMS-condition yield that for $s \in \mathbb{R}$ and $u \in[0, \beta]$,

$$
\omega_{\mathrm{eq}}\left(\tau^{s}(A) \tau^{\mathrm{i} u}(B)\right)=\omega_{\mathrm{eq}}\left(\tau^{-s}(A) \tau^{\mathrm{i} \beta_{\mathrm{eq}}-\mathrm{i} u}(B)\right),
$$

and so

$$
\frac{1}{\beta_{\text {eq }}} \int_{0}^{\beta_{\text {eq }}}\left[\int_{0}^{t} \omega_{\text {eq }}\left(\tau^{s}(A) \tau^{\mathrm{i} u}(B)\right) \mathrm{d} s\right] \mathrm{d} u=\frac{1}{2 \beta_{\text {eq }}} \int_{0}^{\beta_{\text {eq }}}\left[\int_{-t}^{t} \omega_{\text {eq }}\left(A \tau^{s+\mathrm{i} u}(B)\right) \mathrm{d} s\right] \mathrm{d} u .
$$

Since the integral of the function $z \mapsto \omega_{\text {eq }}\left(A \tau^{z}(B)\right)$ over the boundary of the rectangle with vertices $-t, t, t+$ $\mathrm{i} u,-t+\mathrm{i} u$ is zero, we have

$$
\frac{1}{\beta_{\mathrm{eq}}} \int_{0}^{\beta_{\mathrm{eq}}}\left[\int_{0}^{t} \omega_{\mathrm{eq}}\left(\tau^{s}(A) \tau^{\mathrm{i} u}(B)\right) \mathrm{d} s\right] \mathrm{d} u=\frac{1}{2} \int_{-t}^{t} \omega_{\mathrm{eq}}\left(A \tau^{s}(B)\right) \mathrm{d} s+\frac{1}{2 \beta_{\mathrm{eq}}} \int_{0}^{\beta_{\mathrm{eq}}} R(t, u) \mathrm{d} u,
$$

where

$$
R(t, u)=\mathrm{i} \int_{0}^{u}\left[\omega_{\text {eq }}\left(A \tau^{t+\mathrm{i} y}(B)\right)-\omega_{\text {eq }}\left(A \tau^{-t+\mathrm{i} y}(B)\right)\right] \mathrm{d} y .
$$

Assumption (A2) implies that

$$
\lim _{t \rightarrow+\infty} \omega_{\text {eq }}\left(A \tau^{ \pm t+\mathrm{i} y}(B)\right)=\omega_{\mathrm{eq}}(A) \omega_{\mathrm{eq}}(B),
$$

and the dominated convergence theorem yields

$$
\lim _{t \rightarrow+\infty} \sup _{0 \leq u \leq \beta}|R(t, u)|=0 .
$$

This fact and the formula (4.18) yield the statement.

In the next subsection we state our main results concerning the Green-Kubo formulas. 


\subsection{The Green-Kubo formulas}

As already mentioned, our main technical result concerns the differentiability, at $(0,0)$ and for finite $t$ of the function $(X, Y) \mapsto \omega_{X, Y}\left(\tau^{t}(A)\right)$. The resulting finite time linear response formula is the content of the next Theorem. We set

$$
\begin{aligned}
\mathcal{O}_{\vartheta, \mathrm{R}} & =\mathcal{O}_{\vartheta} \cap \operatorname{Dom}\left(\delta_{\mathrm{R}}\right) \cap \operatorname{Dom}\left(\xi_{\mathrm{R}}\right), \\
\mathcal{O}_{\vartheta, \mathrm{R}, \mathrm{c}} & =\mathcal{O}_{\vartheta, \mathrm{R}} \cap \mathcal{C} .
\end{aligned}
$$

Theorem 4.2 Suppose that Assumptions (A1) and (A5) hold and let $A \in \mathcal{O}_{\vartheta, \mathrm{R}, \mathrm{c}}$. Then for all $t \in \mathbb{R}$ the function

$$
(X, Y) \mapsto \omega_{X, Y}\left(\tau^{t}(A)\right),
$$

is differentiable at $(0,0)$ and

$$
\begin{aligned}
& \left.\partial_{X} \omega_{X, Y}\left(\tau^{t}(A)\right)\right|_{X=Y=0}=\mathfrak{L}(A, \Phi, t), \\
& \left.\partial_{Y} \omega_{X, Y}\left(\tau^{t}(A)\right)\right|_{X=Y=0}=\mathfrak{L}(A, \mathcal{J}, t) .
\end{aligned}
$$

We will prove Theorem 4.2 in Subsection 4.3. The next two theorems are consequence of Theorem 4.2, definition of the regular observable, and Proposition 4.1.

Theorem 4.3 Suppose that Assumptions (A1), (A3) and (A5) hold.

(1) Let $A \in \mathcal{O}_{\vartheta, \mathrm{R}, \mathrm{c}}$ be a regular observable. Then

$$
\begin{aligned}
& \left.\partial_{X} \omega_{X, Y,+}(A)\right|_{X=Y=0}=\mathfrak{L}(A, \Phi), \\
& \left.\partial_{Y} \omega_{X, Y,+}(A)\right|_{X=Y=0}=\mathfrak{L}(A, \mathcal{J}) .
\end{aligned}
$$

(2) If in addition (A2) and (A4) hold and $A \in \mathcal{O}_{\vartheta, \mathrm{R}}$ is a regular self-adjoint observable such that $\Theta(A)=-A$, then

$$
\begin{aligned}
& \left.\partial_{X} \omega_{X, Y,+}(A)\right|_{X=Y=0}=\mathcal{L}(A, \Phi), \\
& \left.\partial_{Y} \omega_{X, Y,+}(A)\right|_{X=Y=0}=\mathcal{L}(A, \mathcal{J}) .
\end{aligned}
$$

Theorem 4.4 Suppose that Assumptions (A1), (A3) and (A5) hold and that $\Phi, \mathcal{J}$ are regular observables in $\operatorname{Dom}\left(\delta_{\mathrm{R}}\right) \cap \operatorname{Dom}\left(\xi_{\mathrm{R}}\right)$. Then the formulas (4.17) hold. If in addition (A2) and (A4) hold, then the formulas (4.15) and (4.16) hold.

Theorem 4.2 was proven in [JOP1] in the case $\mu_{\mathrm{eq}}=0, Y=0$. The technical extensions of the proofs in [JOP1] needed to accommodate charge fluxes are relatively minor and are discussed in the next section.

\subsection{Proof of Theorem 4.2}

We will freely use the notation introduced in Subsection 3.1.

Lemma 4.5 (a) The group $\alpha_{0,0}$ preserves $\operatorname{Dom}\left(\delta_{\mathrm{R}}\right) \cap \operatorname{Dom}\left(\xi_{\mathrm{R}}\right)$ and for $A \in \operatorname{Dom}\left(\delta_{\mathrm{R}}\right) \cap \operatorname{Dom}\left(\xi_{\mathrm{R}}\right)$ the functions

$$
\mathbb{R} \ni t \mapsto \delta_{\mathrm{R}}\left(\alpha_{0,0}^{t}(A)\right), \quad \mathbb{R} \ni t \mapsto \xi_{\mathrm{R}}\left(\alpha_{0,0}^{t}(A)\right),
$$


are norm continuous.

(b) For all $t \in \mathbb{R}$ and $A \in \operatorname{Dom}\left(\delta_{\mathrm{R}}\right) \cap \operatorname{Dom}\left(\xi_{\mathrm{R}}\right)$,

$$
\alpha_{X, Y}^{t}(A)-\alpha_{0,0}^{t}(A)=-\frac{X}{\beta_{\text {eq }}} \int_{0}^{t} \alpha_{X, Y}^{t-s}\left(\delta_{\mathrm{R}}\left(\alpha_{0,0}^{s}(A)\right)\right) \mathrm{d} s-\frac{Y}{\beta_{\text {eq }}} \int_{0}^{t} \alpha_{X, Y}^{t-s}\left(\xi_{\mathrm{R}}\left(\alpha_{0,0}^{s}(A)\right)\right) \mathrm{d} s .
$$

(c) For all $t \in \mathbb{R}$ and $A \in \mathcal{O}$,

$$
\lim _{(X, Y) \rightarrow(0,0)}\left\|\alpha_{X, Y}^{t}(A)-\alpha_{0,0}^{t}(A)\right\|=0
$$

(d) For all $A \in \mathcal{O}$,

$$
\lim _{(X, Y) \rightarrow(0,0)} \omega_{X, Y}(A)=\omega_{\mathrm{eq}}(A) .
$$

Proof. To simplify notation let us set $\alpha_{0}=\alpha_{0,0,0}$ and $\alpha=\alpha_{0,0}$. We shall use the identity

$$
\alpha^{t}(A)=\Gamma_{t} \alpha_{0}^{t}(A) \Gamma_{t}^{*},
$$

where $\Gamma_{t} \in \mathcal{O}$ is a family of unitary elements defined by

$$
\Gamma_{t}=\mathbb{1}+\sum_{n \geq 1}(\mathrm{i} t)^{n} \int_{0 \leq s_{n} \leq \cdots s_{1} \leq 1} \alpha_{0}^{t s_{n}}(V) \cdots \alpha_{0}^{t s_{1}}(V) \mathrm{d} s_{1} \cdots \mathrm{d} s_{n},
$$

see Proposition 5.4.1 in [BR2]. Since $V \in \operatorname{Dom}\left(\delta_{\mathrm{R}}\right) \cap \operatorname{Dom}\left(\xi_{\mathrm{R}}\right)$, one easily shows that $\Gamma_{t} \in \operatorname{Dom}\left(\delta_{\mathrm{R}}\right) \cap$ $\operatorname{Dom}\left(\xi_{\mathrm{R}}\right)$ and that

$$
\begin{aligned}
& \delta_{\mathrm{R}}\left(\Gamma_{t}\right)=\sum_{n \geq 1}(\mathrm{i} t)^{n} \int_{0 \leq s_{n} \leq \cdots s_{1} \leq 1} \sum_{j} \alpha_{0}^{t s_{n}}(V) \cdots \alpha_{0}^{t s_{j}}\left(\delta_{\mathrm{R}}(V)\right) \cdots \alpha_{0}^{t s_{1}}(V) \mathrm{d} s_{1} \cdots \mathrm{d} s_{n}, \\
& \xi_{\mathrm{R}}\left(\Gamma_{t}\right)=\sum_{n \geq 1}(\mathrm{i} t)^{n} \int_{0 \leq s_{n} \leq \cdots s_{1} \leq 1} \sum_{j} \alpha_{0}^{t s_{n}}(V) \cdots \alpha_{0}^{t s_{j}}\left(\xi_{\mathrm{R}}(V)\right) \cdots \alpha_{0}^{t s_{1}}(V) \mathrm{d} s_{1} \cdots \mathrm{d} s_{n} .
\end{aligned}
$$

These two formulas yield that the functions

$$
t \mapsto \delta_{\mathrm{R}}\left(\Gamma_{t}\right), \quad t \mapsto \xi_{\mathrm{R}}\left(\Gamma_{t}\right),
$$

are norm continuous. Finally, the identities

$$
\begin{aligned}
& \delta_{\mathrm{R}}\left(\alpha^{t}(A)\right)=\delta_{\mathrm{R}}\left(\Gamma_{t}\right) \alpha_{0}^{t}(A) \Gamma_{t}^{*}+\Gamma_{t} \alpha_{0}^{t}\left(\delta_{\mathrm{R}}(A)\right) \Gamma_{t}^{*}+\Gamma_{t} \alpha_{0}^{t}(A) \delta_{\mathrm{R}}\left(\Gamma_{t}^{*}\right), \\
& \xi_{\mathrm{R}}\left(\alpha^{t}(A)\right)=\xi_{\mathrm{R}}\left(\Gamma_{t}\right) \alpha_{0}^{t}(A) \Gamma_{t}^{*}+\Gamma_{t} \alpha_{0}^{t}\left(\xi_{\mathrm{R}}(A)\right) \Gamma_{t}^{*}+\Gamma_{t} \alpha_{0}^{t}(A) \xi_{\mathrm{R}}\left(\Gamma_{t}^{*}\right),
\end{aligned}
$$

yield Part (a).

If $A \in \operatorname{Dom}\left(\delta_{\mathrm{L}}\right) \cap \operatorname{Dom}\left(\delta_{\mathrm{R}}\right) \cap \operatorname{Dom}\left(\xi_{\mathrm{L}}\right) \cap \operatorname{Dom}\left(\xi_{\mathrm{R}}\right)$, then

$$
\frac{\mathrm{d}}{\mathrm{d} t} \alpha_{X, Y}^{-t} \circ \alpha^{t}(A)=\frac{X}{\beta_{\mathrm{eq}}} \alpha_{X, Y}^{-t}\left(\delta_{\mathrm{R}}\left(\alpha^{t}(A)\right)\right)+\frac{Y}{\beta_{\mathrm{eq}}} \alpha_{X, Y}^{-t}\left(\xi_{\mathrm{R}}\left(\alpha^{t}(A)\right)\right),
$$

and (b) follows. The case $A \in \operatorname{Dom}\left(\delta_{\mathrm{R}}\right) \cap \operatorname{Dom}\left(\xi_{\mathrm{R}}\right)$ is handled by approximating $A$ with the sequence

$$
A_{j}=\frac{j}{\pi} \int_{\mathbb{R}^{2}} \mathrm{e}^{-j\left(t^{2}+s^{2}\right)} \tau_{\mathrm{L}}^{t} \circ \vartheta_{\mathrm{L}}^{s}(A) \mathrm{d} t \mathrm{~d} s
$$

see the proof of Lemma 3.3 in [JOP1].

Since $\operatorname{Dom}\left(\delta_{\mathrm{R}}\right) \cap \operatorname{Dom}\left(\xi_{\mathrm{R}}\right)$ is dense in $\mathcal{O}$, (b) implies (c). The proof of (d) is the same as the proof of Lemma 3.4 in [JOP1]. 
Lemma 4.6 Let $A \in \mathcal{O}_{\vartheta, \mathrm{R}, \mathrm{c}}$. Then for all $t \in \mathbb{R}$ the function

$$
(X, Y) \mapsto \omega_{X, Y}\left(\tau^{t}(A)\right),
$$

is differentiable at $(0,0)$, and

$$
\begin{aligned}
& \left.\partial_{X} \omega_{X, Y}\left(\tau^{t}(A)\right)\right|_{X=Y=0}=\frac{1}{\beta_{\mathrm{eq}}} \int_{0}^{t} \omega_{\mathrm{eq}}\left(\delta_{\mathrm{R}}\left(\tau^{s}(A)\right)\right) \mathrm{d} s \\
& \left.\partial_{Y} \omega_{X, Y}\left(\tau^{t}(A)\right)\right|_{X=Y=0}=\frac{1}{\beta_{\mathrm{eq}}} \int_{0}^{t} \omega_{\mathrm{eq}}\left(\xi_{\mathrm{R}}\left(\tau^{s}(A)\right)\right) \mathrm{d} s .
\end{aligned}
$$

Proof. Since $A$ is a centered observable and $\omega_{X, Y}$ is $\alpha_{X, Y}$-invariant, we have that $\omega_{X, Y}\left(\alpha_{X, Y}^{t}(A)\right)=0$ for all $t$. Since $\alpha_{0,0}=\tau$ on $\mathcal{O}_{\vartheta}$, we have that $\omega_{X, Y}\left(\alpha_{0,0}^{t}(A)\right)=\omega_{X, Y}\left(\tau^{t}(A)\right)$ and $\omega_{0,0}\left(\tau^{t}(A)\right)=\omega_{\text {eq }}\left(\tau^{t}(A)\right)=0$ for all $t$. These observations and Part (b) of Lemma 4.5 imply

$$
\omega_{X, Y}\left(\tau^{t}(A)\right)-\omega_{0,0}\left(\tau^{t}(A)\right)=\frac{X}{\beta_{\mathrm{eq}}} \int_{0}^{t} \omega_{X, Y}\left(\delta_{\mathrm{R}}\left(\tau^{s}(A)\right)\right) \mathrm{d} s+\frac{Y}{\beta_{\mathrm{eq}}} \int_{0}^{t} \omega_{X, Y}\left(\xi_{\mathrm{R}}\left(\tau^{s}(A)\right)\right) \mathrm{d} s .
$$

This relation, Lemma 4.5, and dominated convergence yield the statement.

Lemma 4.7 Assume that $A \in \mathcal{O}_{\vartheta, \mathrm{R}}$. Then

$$
\begin{aligned}
& \omega_{\mathrm{eq}}\left(\delta_{\mathrm{R}}(A)\right)=\int_{0}^{\beta_{\mathrm{eq}}} \omega_{\mathrm{eq}}\left(A \tau^{\mathrm{i} s}(\Phi)\right) \mathrm{d} s, \\
& \omega_{\mathrm{eq}}\left(\xi_{\mathrm{R}}(A)\right)=\int_{0}^{\beta_{\mathrm{eq}}} \omega_{\mathrm{eq}}\left(A \tau^{\mathrm{i} s}(\mathcal{J})\right) \mathrm{d} s .
\end{aligned}
$$

Proof. This lemma is the central and technically most demanding step of the argument. Fortunately, its proof is identical to the proof of Lemma 3.6 in [JOP1]. This follows from the fact that $A, V, \Phi, \mathcal{J} \in \mathcal{O}_{\vartheta}$ and that $\omega_{\text {eq }}\left\lceil\mathcal{O}_{\vartheta}\right.$ is a $\left(\tau, \beta_{\text {eq }}\right)$-KMS state.

Theorem 4.2 is an immediate consequence of Lemmas 4.6 and 4.7.

\section{Some generalizations}

Although we have restricted ourselves in this note to two coupled quantum dynamical systems, the model, the framework and all our results have a straightforward extension to the case of $M$ systems. Let $\beta_{\text {eq }}$ and $\mu_{\text {eq }}$ be the reference (equilibrium) values of the inverse temperature and chemical potential. For $j=1, \ldots, M$ let $\left(\mathcal{O}_{j}, \tau_{j}, \omega_{j, \beta_{j} \mu_{j}}\right)$ be quantum dynamical systems with gauge groups $\vartheta_{j}$ where $\omega_{j}$ is a $\left(\tau_{j}, \vartheta_{j}, \beta_{j}, \mu_{j}\right)$-KMS state. We denote by $\delta_{j}$ and $\xi_{j}$ the generators of $\tau_{j}$ and $\vartheta_{j}$. Assumption (A1) is replaced with

(G1) The reference states of the $j$-th system are parametrized by $\beta_{j} \in I_{\epsilon}\left(\beta_{\text {eq }}\right)$ and $\mu_{j} \in I_{\epsilon}\left(\mu_{\text {eq }}\right)$ and $\omega_{j, \beta_{j} \mu_{j}}$ is the unique $\left(\tau_{j}, \vartheta_{j}, \beta_{j}, \mu_{j}\right)$-KMS state on $\mathcal{O}_{j}$. 
Let $\mathcal{O}=\mathcal{O}_{1} \otimes \cdots \otimes \mathcal{O}_{M}, \tau_{0}=\tau_{1} \otimes \cdots \otimes \tau_{M}, \vartheta=\vartheta_{1} \otimes \cdots \otimes \vartheta_{M}$. The algebra $\mathcal{O}_{\vartheta}$ is again defined by (2.1). The pair $\left(\mathcal{O}, \tau_{0}\right)$ describes the uncoupled joint system. Let $V \in \mathcal{O}_{\vartheta}$ be a self-adjoint perturbation and $\tau$ the perturbed $C^{*}$-dynamics. The coupled joint system is described by $(\mathcal{O}, \tau)$. The thermodynamical forces are

$$
X_{j}=\beta_{\mathrm{eq}}-\beta_{j}, \quad Y_{j}=\beta_{j} \mu_{j}-\beta_{\mathrm{eq}} \mu_{\mathrm{eq}} .
$$

We set $X=\left(X_{1}, \ldots, X_{M}\right), Y=\left(Y_{1}, \ldots, Y_{M}\right)$. The reference state is $\omega_{X, Y, 0}=\omega_{1, \beta_{1} \mu_{1}} \otimes \cdots \omega_{M, \beta_{M} \mu_{M}} \cdot \omega_{X, Y, 0}$ is the unique $\beta_{\mathrm{eq}}$-KMS state for the $C^{*}$-dynamics

$$
\alpha_{X, Y, 0}^{t}=\left[\tau_{1}^{\beta_{1} t / \beta_{\mathrm{eq}}} \circ \vartheta_{1}^{-\mu_{1} \beta_{1} t / \beta_{\mathrm{eq}}}\right] \otimes \cdots \otimes\left[\tau_{M}^{\beta_{M} t / \beta_{\mathrm{eq}}} \circ \vartheta_{M}^{-\mu_{M} \beta_{M} t / \beta_{\mathrm{eq}}}\right] .
$$

Let $\delta_{X, Y, 0}$ be the generator of $\alpha_{X, Y, 0}$ and $\delta_{X, Y}=\delta_{X, Y, 0}+\mathrm{i}[V, \cdot]$. Let $\alpha_{X, Y}$ be the $C^{*}$-dynamics generated by $\delta_{X, Y}$ and let $\omega_{X, Y}$ be the $\left(\alpha_{X, Y}, \beta_{\text {eq }}\right)$-KMS state obtained from $\omega_{X, Y, 0}$ by Araki's perturbation theory. This completes the setup of the model. Note that the state $\omega_{\mathrm{eq}} \equiv \omega_{0,0}$ is the unique $\left(\tau, \vartheta, \beta_{\mathrm{eq}}, \mu_{\mathrm{eq}}\right)$-KMS state on $\mathcal{O}$. Assumptions (G2) has the same formulation as Assumption (A2) and Assumptions (A3), (A4) and (A5) are replaced with:

(G3) For all $X, Y \in I_{\epsilon}^{M}$ there exists a state $\omega_{X, Y,+}$ on $\mathcal{O}_{\vartheta}$ such that for all $A \in \mathcal{O}_{\vartheta}$,

$$
\lim _{t \rightarrow+\infty} \omega_{X, Y}\left(\tau^{t}(A)\right)=\omega_{X, Y,+}(A) .
$$

(G4) There exists a time-reversal $\Theta$ of $\left(\mathcal{O}, \tau_{0}\right)$ such that $\Theta(V)=V$ and

$$
\Theta \circ \tau_{j}^{t}=\tau_{j}^{-t} \circ \Theta, \quad \Theta \circ \vartheta_{j}^{t}=\vartheta_{j}^{-t} \circ \Theta
$$

for all $j$.

(G5) $V \in \operatorname{Dom}\left(\delta_{j}\right) \cap \operatorname{Dom}\left(\xi_{j}\right)$ for all $j$.

The observables associated to the heat and charge flux out of the $j$-th system are

$$
\Phi_{j}=\delta_{j}(V), \quad \mathcal{J}_{j}=\xi_{j}(V) .
$$

It immediately follows that

$$
\sum_{j=1}^{M} \omega_{X, Y,+}\left(\Phi_{j}\right)=0 \text { and } \sum_{j=1}^{M} \omega_{X, Y,+}\left(\mathcal{J}_{j}\right)=0
$$

which are respectively the first law of thermodynamics (conservation of energy) and charge conservation. The entropy balance equation reads

$$
\operatorname{Ent}\left(\omega_{X, Y} \circ \tau^{t} \mid \omega_{X, Y}\right)=-\sum_{j=1}^{M} X_{j} \int_{0}^{t} \omega_{X, Y}\left(\tau^{s}\left(\Phi_{j}\right)\right) \mathrm{d} s-\sum_{j=1}^{M} Y_{j} \int_{0}^{t} \omega_{X, Y}\left(\tau^{s}\left(\mathcal{J}_{j}\right)\right) \mathrm{d} s
$$

and in particular the second law holds:

$$
\operatorname{Ep}\left(\omega_{X, Y,+}\right)=\sum_{j=1}^{M} X_{j} \omega_{X, Y,+}\left(\Phi_{j}\right)+\sum_{j=1}^{M} Y_{j} \omega_{X, Y,+}\left(\mathcal{J}_{j}\right) \geq 0
$$

The definition of the centered observable is the same as in Subsection 3.7. We set

$$
\begin{aligned}
\hat{\mathcal{O}}_{\vartheta} & =\left(\cap_{j=1}^{M} \operatorname{Dom}\left(\delta_{j}\right)\right) \cap\left(\cap_{j=1}^{M} \operatorname{Dom}\left(\xi_{j}\right)\right) \cap \mathcal{O}_{\vartheta}, \\
\hat{\mathcal{O}}_{\vartheta, \mathrm{c}} & =\hat{\mathcal{O}}_{\vartheta} \cap \mathcal{C} .
\end{aligned}
$$


If $V \in \hat{\mathcal{O}}_{\vartheta}$, then $\Phi_{j}, \mathcal{J}_{j} \in \hat{\mathcal{O}}_{\vartheta, \mathrm{c}}$ for all $j$ (after obvious notational changes, Proposition 3.2 applies directly to the model consider in this section).

Theorem 4.2 is replaced with:

Theorem 5.1 Suppose that Assumptions (G1) and (G5) hold and let $A \in \hat{\mathcal{O}}_{\vartheta, c}$. Then for all $t \in \mathbb{R}$ the function

$$
(X, Y) \mapsto \omega_{X, Y}\left(\tau^{t}(A)\right),
$$

is differentiable at $(0,0)$ and

$$
\begin{gathered}
\left.\partial_{X_{j}} \omega_{X, Y}\left(\tau^{t}(A)\right)\right|_{X=Y=0}=\mathfrak{L}\left(A, \Phi_{j}, t\right), \\
\left.\partial_{Y_{j}} \omega_{X, Y}\left(\tau^{t}(A)\right)\right|_{X=Y=0}=\mathfrak{L}\left(A, \mathcal{J}_{j}, t\right) .
\end{gathered}
$$

The definition of the regular observable is the same as before, and we have:

Theorem 5.2 Suppose that Assumptions (G1), (G3) and (G5) hold.

(1) Let $A \in \hat{\mathcal{O}}_{\vartheta, \mathrm{c}}$ be a regular observable. Then

$$
\begin{aligned}
& \left.\partial_{X_{j}} \omega_{X, Y,+}(A)\right|_{X=Y=0}=\mathfrak{L}\left(A, \Phi_{j}\right), \\
& \left.\partial_{Y_{j}} \omega_{X, Y,+}(A)\right|_{X=Y=0}=\mathfrak{L}\left(A, \mathcal{J}_{j}\right) .
\end{aligned}
$$

(2) If in addition (G2) and (G4) hold and $A \in \hat{\mathcal{O}}_{\vartheta}$ is a regular self-adjoint observable such that $\Theta(A)=-A$, then

$$
\begin{aligned}
& \left.\partial_{X_{j}} \omega_{X, Y,+}(A)\right|_{X=Y=0}=\mathcal{L}\left(A, \Phi_{j}\right), \\
& \left.\partial_{Y_{j}} \omega_{X, Y,+}(A)\right|_{X=Y=0}=\mathcal{L}\left(A, \mathcal{J}_{j}\right) .
\end{aligned}
$$

Theorem 5.3 Suppose that (G1), (G3) and (G5) hold and that $\Phi_{j}, \mathcal{J}_{j}$ are regular observables in $\operatorname{Dom}\left(\delta_{j}\right) \cap$ $\operatorname{Dom}\left(\xi_{j}\right)$. Then:

(1) The kinetic transport coefficients

$$
\begin{aligned}
& L_{\mathrm{hh}}^{k j}=\left.\partial_{X_{j}} \omega_{X, Y,+}\left(\Phi_{k}\right)\right|_{X=Y=0}, \\
& L_{\mathrm{hc}}^{k j}=\left.\partial_{Y_{j}} \omega_{X, Y,+}\left(\Phi_{k}\right)\right|_{X=Y=0}, \\
& L_{\mathrm{ch}}^{k j}=\left.\partial_{X_{j}} \omega_{X, Y,+}\left(\mathcal{J}_{k}\right)\right|_{X=Y=0}, \\
& L_{\mathrm{cc}}^{k j}=\left.\partial_{Y_{j}} \omega_{X, Y,+}\left(\mathcal{J}_{k}\right)\right|_{X=Y=0},
\end{aligned}
$$

satisfy

$$
\begin{aligned}
L_{\mathrm{hh}}^{k j} & =\mathfrak{L}\left(\Phi_{k}, \Phi_{j}\right), \\
L_{\mathrm{hc}}^{k j} & =\mathfrak{L}\left(\Phi_{k}, \mathcal{J}_{j}\right), \\
L_{\mathrm{ch}}^{k j} & =\mathfrak{L}\left(\mathcal{J}_{k}, \Phi_{j}\right), \\
L_{\mathrm{cc}}^{k j} & =\mathfrak{L}\left(\mathcal{J}_{k}, \mathcal{J}_{j}\right) .
\end{aligned}
$$


Assume in addition that (G2) and (G4) hold. Then

(2) The Green-Kubo formulas hold:

$$
\begin{aligned}
& L_{\mathrm{hh}}^{k j}=\mathcal{L}\left(\Phi_{k}, \Phi_{j}\right), \\
& L_{\mathrm{hc}}^{k j}=\mathcal{L}\left(\Phi_{k}, \mathcal{J}_{j}\right), \\
& L_{\mathrm{ch}}^{k j}=\mathcal{L}\left(\mathcal{J}_{k}, \Phi_{j}\right), \\
& L_{\mathrm{cc}}^{k j}=\mathcal{L}\left(\mathcal{J}_{k}, \mathcal{J}_{j}\right) .
\end{aligned}
$$

(3) The Onsager reciprocity relations hold:

$$
\begin{aligned}
& L_{\mathrm{hh}}^{k j}=L_{\mathrm{hh}}^{j k}, \\
& L_{\mathrm{cc}}^{k j}=L_{\mathrm{cc}}^{j k}, \\
& L_{\mathrm{hc}}^{k j}=L_{\mathrm{ch}}^{j k} .
\end{aligned}
$$

The remark after Theorem 4.4 applies to Theorems 5.2 and 5.3.

In the literature one often considers a special case, described in the introduction, where one of the quantum dynamical systems, say $\left(\mathcal{O}_{1}, \tau_{1}, \omega_{1, \beta_{1} \mu_{1}}\right)$, is finite dimensional and plays a role of a "small" quantum system $\mathcal{S}$ coupled to reservoirs described by $\left(\mathcal{O}_{j}, \tau_{j}, \omega_{j, \beta_{j} \mu_{j}}\right), j \geq 2$. Such systems are one of the basic paradigms of nonequilibrium quantum statistical mechanics and have played an important role in the historical development of the subject. With regard to the algebraic approach described in this note, the only additional feature of these models is vanishing of heat and charge fluxes out of the small system: $\omega_{X, Y,+}\left(\Phi_{1}\right)=\omega_{X, Y,+}\left(\mathcal{J}_{1}\right)=0$.

Many other generalizations are possible and it appears difficult to have a unified framework which covers all cases of physical interest. The Electronic Black Box Models studied in [AJPP1, AJPP2, JOPP] are examples of open quantum systems which do not fit directly into the class of models described here (the non-interacting coupled system is not a tensor product of the individual subsystems). However, the changes needed to apply our results to these models are elementary. One may also consider $W^{*}$-dynamical systems instead of $C^{*}$-dynamical systems and unbounded interactions which are only affiliated to the algebra of observables. The models where such generalization is necessary involve free bosonic reservoirs (a well-known example is the spin-boson model). One may also consider time-dependent interactions (see [Ru1, JP3, JP4, ASF]). Another possible generalization involves more general gauge groups. The important point is that although all such generalizations may require some adjustment of technique and presentation, they bring nothing conceptually new.

\section{References}

[Ar1] Araki, H.: Relative entropy of states of von Neumann algebras. Publ. Res. Inst. Math. Sci. Kyoto Univ. 11, 809 (1975/76).

[Ar2] Araki, H.: Relative entropy of states of von Neumann algebras II. Publ. Res. Inst. Math. Sci. Kyoto Univ. 13, 173 (1977/78).

[ASF] Abou-Salem, W.K., Fröhlich, J.: Adiabatic Theorems and Reversible Isothermal Processes. Lett. Math. Phys., in press.

[AH] Araki, H., Ho, T.G: Asymptotic time evolution of a partitioned infinite two-sided isotropic $X Y$-chain. Tr. Mat. Inst. Steklova, 228 Probl. Sovrem. Mat. Fiz., 203, (2000); translation in Proc. Steklov Inst. Math. 228, 191, (2000).

[AP] Aschbacher, W., Pillet, C-A.: Non-equilibrium steady states of the $X Y$ chain. J. Stat. Phys. 12, 1153 (2003). 
[AJPP1] Aschbacher, W., Jakšić, V., Pautrat, Y., Pillet, C.-A.: Topics in non-equilibrium quantum statistical mechanics. In Open Quantum Systems III. S. Attal, A. Joye, C.-A. Pillet editors. Lecture Notes in Mathematics 1882, Springer, New York (2006).

[AJPP2] Aschbacher, W., Jakšić, V., Pautrat, Y., Pillet, C.-A.: Transport properties of ideal Fermi gases (in preparation).

[AM] Aizenstadt, V.V., Malyshev, V.A.: Spin interaction with an ideal Fermi gas. J. Stat. Phys. 48, 51 (1987).

[BM] Botvich, D.D., Malyshev, V.A.: Unitary equivalence of temperature dynamics for ideal and locally perturbed Fermi Gas. Commun. Math. Phys. 61, 209 (1978).

[BR1] Bratteli, O, Robinson D. W.: Operator Algebras and Quantum Statistical Mechanics 1. Springer-Verlag, Berlin (1987).

[BR2] Bratteli, O, Robinson D. W.: Operator Algebras and Quantum Statistical Mechanics 2. Second edition, SpringerVerlag, Berlin (1996).

[Da] Davies, E.B.: Markovian master equations. Commun. Math. Phys. 39, 91 (1974).

[DGM] De Groot, S.R., Mazur, P.: Non-Equilibrium Thermodynamics. North-Holland, Amsterdam (1969).

[DJP] Dereziński, J., Jakšić, V., Pillet, C.-A.: Perturbation theory of $W^{*}$-dynamics, KMS-states and Liouvillean. Rev. Math. Phys. 15, 447 (2003).

[Don] Donald, M.J.: Relative Hamiltonians which are not bounded from above. J. Func. Anal. 91, 143 (1990).

[EM] Evans, D.J., Morriss, G.P.: Statistical Mechanics of Nonequilibrium Liquids. Academic Press, London (1990).

[FMU] Fröhlich, J., Merkli, M., Ueltschi, D.: Dissipative transport: thermal contacts and tunneling junctions. Ann. Henri Poincaré 4, 897 (2004).

[JOP1] Jakšić, V., Ogata, Y., Pillet, C.-A.: The Green-Kubo formula and Onsager reciprocity relations in quantum statistical mechanics. Commun. Math. Phys, in press.

[JOP2] Jakšić, V., Ogata, Y., Pillet, C.-A.: The Green-Kubo formula for the spin-fermion model. Submitted.

[JOP3] Jakšić, V., Ogata, Y., Pillet, C.-A.: In preparation.

[JOPP] Jakšić, V., Ogata, Y., Pautrat, Y., Pillet, C.-A.: In preparation.

[JP1] Jakšić, V., Pillet, C-A.: On entropy production in quantum statistical mechanics. Commun. Math. Phys. 217, 285 (2001).

[JP2] Jakšić, V., Pillet, C.-A.: Non-equilibrium steady states for finite quantum systems coupled to thermal reservoirs. Commun. Math. Phys. 226, 131 (2002).

[JP3] Jakšić, V., Pillet, C.-A.: Mathematical theory of non-equilibrium quantum statistical mechanics. J. Stat. Phys. 108, 787 (2002).

[JP4] Jakšić, V., Pillet, C.-A.: A note on the entropy production formula. Contemp. Math. 327, 175 (2003).

[KTH] Kubo, R., Toda, M., Hashitsune, N.: Statistical Physics II. Second edition, Springer-Verlag, Berlin (1991).

[LeSp] Lebowitz, J., Spohn, H.: Irreversible thermodynamics for quantum systems weakly coupled to thermal reservoirs. Adv. Chem. Phys. 39, 109 (1978).

[RB] Rey-Bellet, L.: Open Classical Systems. In Open Quantum Systems II. S. Attal, A. Joye, C.-A. Pillet editors. Lecture Notes in Mathematics 1881, Springer, New York (2006).

[Ru1] Ruelle, D.: Natural nonequilibrium states in quantum statistical mechanics. J. Stat. Phys. 98, 57 (2000).

[Ru2] Ruelle, D.: Entropy production in quantum spin systems. Commun. Math. Phys. 224, 3 (2001).

[Ru3] Ruelle, D.: Topics in quantum statistical mechanics and operator algebras. Preprint, mp-arc 01-257 (2001).

[Ru4] Ruelle, D.: Smooth dynamics and new theoretical ideas in nonequilibrium statistical mechanics. J. Stat. Phys. 95, 393 (1999). 